\title{
Homocysteine: a novel risk factor for coronary heart disease in UK Indian Asians
}

The population of the UK includes approximately 1.6 million people of Indian Asian descent, most of whom are first or second generation migrants. In UK Indian Asians, mortality from coronary heart disease (CHD) is $40 \%$ higher, ${ }^{1}$ and admission rates with myocardial infarction are twofold higher, ${ }^{2}$ compared to the European white population. The increase in CHD risk is most striking in young men, among whom CHD mortality rates are twice those in Europeans. ${ }^{1}$

The mechanisms underlying increased CHD mortality in UK Indian Asians are not well understood. Population studies show that levels of cigarette smoking, blood pressure, and cholesterol are not consistently raised in Indian Asians, compared to Europeans, ${ }^{3-4}$ indicating that these conventional risk factors do not account for the excess CHD mortality in Indian Asians. ${ }^{3}$ In contrast, diabetes and insulin resistance are more prevalent in Indian Asians than European whites, ${ }^{3-5}$ although their precise contribution to increased CHD risk in Asians remains to be determined.

\section{Raided plasma homocysteine: an emerging risk factor for vascular disease}

Homocysteine is a sulfur containing amino acid, derived from the metabolism of dietary methionine (fig 1). ${ }^{6}$ Homocysteine concentrations are determined by genetic and nutritional factors; mutations in the genes for enzymes involved in homocysteine metabolism, such as the common 5,10-methylenetetrahydrofolate reductase
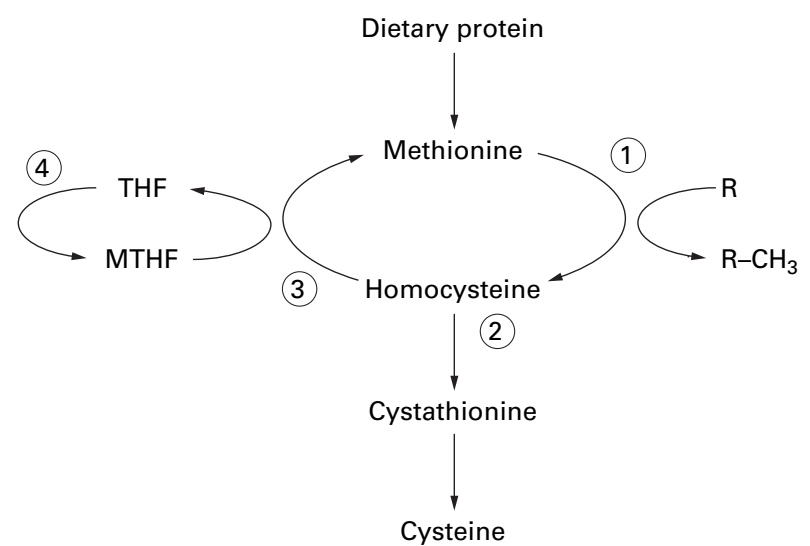

Figure 1 Outline of metabolic pathway for homocysteine. (1) Transmethylation. Conversion of methionine to homocysteine, thereby transferring methyl group to other species (R). (2) Transsulfuration. Irreversible conversion of homocysteine to cysteine: via rate limiting enzyme cystathionine- $\beta$-synthase, with vitamin $B_{6}$ as essential co-factor. (3) Remethylation. Regeneration of methionine from homocysteine: catabolised by methionine synthase, with 5,10-methylene-tetrahydrofolate (MTHF, a form of folic acid) and vitamin $B_{12}$, as essential co-factors. (4) Regeneration of MTHF from tetrahydrofolate (THF), catabolised by enzyme 5,10-methylene-tetrahydrofolate reductase (MTHFR).
(MTHFR) $677 \mathrm{C} \rightarrow \mathrm{T}$ mutation, and deficiencies of vitamins $\mathrm{B}_{6}, \mathrm{~B}_{12}$, and folic acid (essential co-factors for homocysteine metabolism) are associated with raised homocysteine concentrations.

Children with the rare metabolic disorder homocystinuria, who have severe hyperhomocysteinaemia, develop widespread, premature atherosclerosis. ${ }^{7}$ This observation led to the hypothesis that homocysteine might contribute to the development of atherosclerosis in adults. This possibility has now been evaluated in more than 30 studies, involving over 10000 subjects. ${ }^{89}$ The results have been remarkably consistent, with few exceptions. Raised plasma homocysteine is an independent risk factor for peripheral vascular, cerebrovascular, and coronary heart disease, and in prospective studies, homocysteine concentrations of 9 , 15 , and $20 \mu \mathrm{mol} / 1$ predict total mortality ratios of $1.9,2.8$, and 4.5 , respectively. ${ }^{10}$ Homocysteine concentrations exceeding the upper limit of normal $(15 \mu \mathrm{mol} / \mathrm{l})$ are common, and are found in almost $30 \%$ of patients with vascular disease. In North American and European populations, it is estimated that raised homocysteine may contribute to $10 \%$ of population CHD risk. ${ }^{8}$

\section{Homocysteine concentrations in Indian Asians}

Recent studies show that plasma homocysteine concentrations are higher in UK Indian Asians than European whites, ${ }^{11-13}$ and also confirm that homocysteine is a risk factor for CHD in this racial group. ${ }^{11}$ Based upon these findings, it is estimated that elevated homocysteine may contribute to twice as many CHD deaths in UK Asians compared to Europeans. ${ }^{11}$ Among UK Indian Asians, raised homocysteine is accounted for by reduced concentrations of vitamins $B_{12}$ and folate, compared to Europeans, ${ }^{11}$ and not by differences in renal function, ${ }^{14}$ suggesting that nutritional factors may underlie raised plasma homocysteine concentrations in Asians. Reduced intake of vitamin $B_{12}$ has been reported in Indian Asians, and prolonged cooking of vegetables, which is common practice in many Indian Asian households, may destroy up to $90 \%$ of folate content. ${ }^{15-17}$ These observations imply that the increased CHD risk in this racial group may be reduced by dietary supplementation with $\mathrm{B}$ vitamins. In contrast, unlike Europeans, the common MTHFR 677T mutation does not influence homocysteine concentrations in Asians, despite their lower folate concentrations. ${ }^{18}$ In fact, the frequency of homozygosity for MTHFR 677T among UK Indian Asians is less than one third that in European whites. ${ }^{18}$ These findings exclude a role for this mutation underlying increased CHD risk in UK Indian Asians, although do not discount the possibility that novel genetic defects, other than the MTHFR C677T mutation, influence homocysteine metabolism in this racial group. 


\section{Homocysteine: marker or causal agent in vascular disease?}

It remains unknown whether homocysteine has a causal role in the development of atherosclerosis, or is simply a marker for increased vascular risk. ${ }^{6}{ }^{19}$ Evidence to support the former has emerged from studies showing a dynamic and inverse relation between plasma homocysteine and vascular endothelial function. An acute increase in homocysteine is associated with rapid onset vascular endothelial dysfunction - an early manifestation of atherosclerosisand this effect may be mediated by an increase in oxidation stress. ${ }^{20-22}$ Rapid onset endothelial dysfunction can also be demonstrated following physiological increments in plasma homocysteine induced by low dose oral methionine, or dietary animal protein. ${ }^{23}$ These findings are consistent with in vitro reports of a dose and time dependent effect of homocysteine on endothelial cellular function, ${ }^{24-26}$ and suggest that even diet related increments in plasma homocysteine may contribute to the development and progression of atherosclerosis.

Additional evidence to support a causal role for homocysteine in vascular disease comes from studies investigating the effects of homocysteine lowering through $B$ vitamin supplementation. In healthy volunteers, and in patients with CHD, B vitamin supplementation is associated with an improvement in endothelium dependent dilatation, and in serum markers of endothelial injury. ${ }^{27-30}$ Furthermore, among healthy siblings of patients with premature atherosclerosis, homocysteine lowering reduces the occurrence of abnormal exercise tests, consistent with a decreased risk of future atherosclerotic coronary events. ${ }^{31}$ More conclusive evidence may emerge from the results of the large scale randomised, placebo controlled, intervention trials currently in progress, investigating whether homocysteine lowering will reduce cardiovascular events in patients with CHD. These studies are expected to report within the next five years. ${ }^{32}$

\section{Conclusions}

CHD mortality is higher in UK Indian Asians than European whites, and is not accounted for by conventional coronary risk factors. Recent studies show that homocysteine concentrations are higher in Indian Asians than European whites, ${ }_{11}^{112}$ and suggest that homocysteine may contribute to their increased CHD mortality. ${ }^{11}$ These observations imply that the increased CHD risk in this racial group may be reduced by dietary supplementation with B vitamins. However, at present, there are no data to show that lowering homocysteine will reduce major cardiovascular end points. The results of large scale intervention studies with hard end points are awaited with interest.

JOHN C CHAMBERS JASPAL S KOONER

National Heart and Lung Institute,

Imperial College School of Medicine,

Hammersmith Hospital,

Du Cane Road,

London W12 ONN, UK

j.kooner@ic.ac.uk

1 Balarajan R. Ethnicity and variations in mortality from coronary heart disease. Health Trends 1996;28:45-51.
2 Wilkinson P, Sayer J, Laji K, et al. Comparison of case fatality in south Asian and white patients after acute myocardial infarction: observational study. BMF 1996;312:1330-3.

3 McKeigue PM, Shah B, Marmot MG. Relation of central obesity and insulin resistance with high diabetes prevalence and cardiovascular risk in South Asians. Lancet 1991;337:382-6.

4 Bhopal R, Unwin N, White M, et al. Heterogeneity of coronary heart disease risk factors in Indian, Pakistani, Bangladeshi, and European origin populations: cross sectional study. $B M \mathcal{F} 1999 ; 319 \cdot 215-20$

5 McKeigue PM, Ferrie JE, Pierpoint T, et al. Association of early-onset coronary heart disease in South Asian men with glucose intolerance and hyperinsulinemia. Circulation 1993;87:152-61.

6 Hankey JH, Eikelboom JW. Homocysteine and vascular disease. Lancet 1999;354:407-13.

McCully KS. Vascular pathology of homocyst(e)inemia: implications for the development of arteriosclerosis. Am f Pathol 1969;56:111-28.

8 Boushey CJ, Beresford SA, Omenn GS, et al. A quantitative assessment of plasma homocysteine as a risk factor for vascular disease. Probable benefits of increasing folic acid intakes. $7 A M A$ 1995;274:1049-57.

9 Danesh J, Lewington S. Plasma homocysteine and coronary heart disease: systematic review of published epidemiological studies. $f$ Cardiovasc Risk 1998;5:229-32.

10 Nygard O, Nordrehaug JE, Refsum H, et al. Plasma homocysteine levels and mortality in patients with coronary artery disease. N Engl F Med 1997;337: 230-6.

11 Chambers JC, Obeid OA, Refsum H, et al. Plasma homocysteine concentrations and risk of coronary heart disease in UK Indian Asian and European men. Lancet 2000;355:523-7.

12 Anand SS, Yusuf S, Vuksan V, et al. Differences in risk factors, atherosclerosis, and cardiovascular disease between ethnic groups in Canada: the Study of health assessment and risk in ethnic groups (SHARE). Lancet 2000;356: $279-84$.

13 Obeid OA, Mannan N, Perry G, et al. Homocysteine and folate in healthy east London Bangladeshis. Lancet 1998;352:1829-30.

14 Chambers JC, Kooner JS. Lancet 2000;355:2250.

15 Abraham R, Brown MC, North WR, et al. Diets of Asian pregnant women in Harrow: iron and vitamins. Hum Nutr Appl Nutr 1987;41:164-73.

16 Matthews JH, Wood JK. Megaloblastic anaemia in vegetarian Asians. Clin Lab Haematol 1984;6:1-7.

17 Dawson DW, Waters HM. Malnutrition: folate and cobalamin deficiency $\mathrm{Br}$ 7 Biomed Sci 1994;51:221-7.

18 Chambers JC, Ireland $\mathrm{H}$, Thompson $\mathrm{E}$, et al. The methylenetetrahydrofolate reductase $677 \mathrm{C} \rightarrow \mathrm{T}$ mutation, and coronary heart disease risk in UK Indian Asians. Arterioscler Thromb Vasc Biol 2000;20:2448-52.

19 Dudman NP. An alternative view of homocysteine. Lancet 1999;354:2072-

20 Chambers JC, McGregor A, Jean Marie J, et al. Demonstration of rapid onset vascular endothelial dysfunction after hyperhomocysteinemia: an effect reversible with vitamin C therapy. Circulation 1999;99:1156-60.

21 Bellamy MF, McDowell IF, Ramsey MW, et al. Hyperhomocysteinemia after an oral methionine load acutely impairs endothelial function in healthy adults. Circulation 1998;98:1848-52.

22 Kanani PM, Sinkey CA, Browning RL, et al. Role of oxidant stress in endothelial dysfunction produced by experimental hyperhomocysteinaemia in humans. Circulation 1999;100:1161-8.

23 Chambers JC, Obeid OA, Kooner JS. Physiological increments in plasma homocysteine induce vascular endothelial dysfunction in normal human subjects. Arterioscler Thromb Vasc Biol 1999;19:2922-7.

24 Nishinaga M, Ozawa T, Shimada K. Homocysteine, a thrombogenic agent, suppresses anticoagulant heparan sulfate expression in cultured porcine aortic endothelial cells. $\mathcal{F}$ Clin Invest 1993;92:1381-6.

25 Upchurch GR Jr, Welch GN, Fabian AJ, et al. Homocyst(e)ine decreases bioavailable nitric oxide by a mechanism involving glutathione peroxidase. f Biol Chem 1997;272:17012-7.

26 Dudman NP, Temple SE, Guo XW, et al. Homocysteine enhances neutrophil-endothelial interactions in both cultured human cells and rats in vivo. Circ Res 1999;84:409-16

27 Chambers JC, Ueland PM, Obeid OA, et al. Improved vascular endothelial function after oral B vitamins; an effect mediated through reduced concentrations of free plasma homocysteine. Circulation 2000;102:2479-83.

28 Woo KS, Chook P, Lolin YI, et al. Folic acid improves arterial endothelial function in adults with hyperhomocystinemia. I Am Coll Cardiol 1999;34:2002-6.

29 Bellamy MF, McDowell IF, Ramsey MW, et al. Oral folate enhances endothelial function in hyperhomocysteinaemic subjects. Eur f Clin Invest 1999;29:659-62.

30 van den Berg M, Boers GH, Franken DG, et al. Hyperhomocysteinaemia and endothelial dysfunction in young patients with peripheral arterial occlusive disease. Eur f Clin Invest 1995;25:176-81.

31 Vermeulen EG, Stehouwer CD, Twisk JW, et al. Effect of homocysteinelowering treatment with folic acid plus vitamin B6 on progression of subclinical atherosclerosis: a randomised, placebo-controlled trial. Lancet 2000;355:517-22.

32 Clarke R, Collins R. Can dietary supplements with folic acid or vitamin B6 reduce cardiovascular risk? Design of clinical trials to test the homocysteine hypothesis of vascular disease. F Cardiovasc Risk 1998;5:249-55. 